\title{
Reshaping of communicative and cultural codes in the new global digital communicative sphere
}

\author{
Verbitskaya Olga ${ }^{1, *}$ and Lesnikovskaya Ekaterina $^{1}$ \\ ${ }^{1}$ Irkutsk State University, Department of Oriental and Asia-Pacific Regional Studies, 664000, Irkutsk, Russia
}

\begin{abstract}
Today, new global digital communicative space is emerging and consolidating, in which the players - national states, business units, individuals - all struggle to find and secure their place. The new reality that implies communication through global digital platforms seems to be reshaping and redefining the "global village"; it produces and reproduces a new set of cultural symbols and sociocultural codes. The article covers the unique characteristics that the new discourse space has. Special attention is paid to one of the most influential features of the new global digital communicative sphere - the remarkable intensity of the information content. The authors argue that cultural codes and patterns change following the changes in the global communicative sphere.
\end{abstract}

\section{Introduction}

The way our language and our communication work has long depended on a wide range of sociocultural, socioeconomic and sociopolitical issues such as international relations, wars, global and regional sociopolitical agenda, international trade and technological advancement. These bases still exist today; however, there appear new combinations of factors as well as the combinations of familiar challenges with a new reality, new surroundings. For instance, we are currently dealing with COVID-19 pandemic, which is a global biological challenge and a threat penetrating national borders - although this is a kind of phenomenon the humankind has faced a number of times in the course of history; today this global biological threat is combined with current level of digital technologies. This combination of a familiar challenge with a new reality creates a space for modifying both the way people are communicating with one another and the way they are communicating their thoughts, ideas, and messages. Another illustration is the influence of global trade and global business links on the communication and cultural relations - again. Although this has been in place for centuries, today we have transnational corporations that do not remain in the frames of "just business" but have achieved social, political and cultural dominance. Transnational corporations having the budget equaling and even exceeding the budgets of many national states do not only grow as influential figures in the financial and legal space, but also impose sociocultural norms through their corporate cultures and corporate discourse. The phenomenon as such is not new for the humanity but in the new reality it produces and reproduces a new set of cultural symbols and sociocultural codes.
These new combinations of factors that create current specific sociocultural environment seem to be reshaping and redefining the "global village" as we have known it for the last 40 years. The process we are witnessing can be viewed as dividing the power in the digital space. The players - national states, business units, individuals that can reach to a large audience in the digital communicative space (the so-called "influencers") - all struggle to find and secure their space in this new global communicative sphere. This sphere is complicated - on the one hand, consolidated in just a few Internet resources (Twitter, Facebook, Instagram, Youtube), and diverse and loosely organized, on the other. The global space is large (information is unlimited) but the "currency" that is used in the space - people's attention - as we know from economy of attention, is limited. The main tool that is used to influence and control the flows of communication, the content and the values is the language.

\section{The information in the new global digital sphere}

The functioning of the global communicative sphere requires changes in language, and the changes in language define and manifest the traits of the new reality. As D. Bolinger puts it, “...The marvelous thing is that every language has the capacity to take the form that its users require. But it also, through its existing form, helps to shape the requirements..." [1]. In these circumstances, the process of transmission of cultural codes and messages is manifested. The new technologies such as projects on neuronet and artificial intelligence challenge the way we think of the speaker - the subject of communication. We communicate with chat bots and electronic assistants. Texts can be created by AI. In this

Corresponding author: overbitskaya@gmail.com 
case, what happens to the value component of the language and what happens to the cultural codes?

In translating from one language to another, there has always been the human - the bearer of values and cultural codes - the translator (the interpreter). With machine translation, we now have no such subject, thus no value and cultural code transmission, no cultural mediator. These and many other questions have not yet been reflected or discussed in academia and among the public, although they will define the process of the translation (transmission) of cultural codes.

The process of transforming the sociocultural codes seems to be uncontrolled. However, sociologists know that any social process is controlled and managed. Thus, we face the question of who (or what) manages and controls this process. This question is crucial because language is a strategic tool and a weapon: It is the language that ultimately defines the borders of economic, political, sociocultural influence. For instance, the "Russian world" confines to Russianspeaking countries (where usually Russian is not the official language but widely used) and Russian-speaking audience in a number of countries. Language is the main tool that creates sociocultural codes, the DNA of the society.

In this view, what we are dealing with today is a new discursive space that transforms cultural codes. The new discursive space has its unique characteristics such as specific ways of thinking we could call hashtag thinking, frame thinking, clip thinking (clip perception), and it shapes ideas, norms, attitudes, social behavior, political orientation, life views.

The problems that appear in the new digital communicative space can be divided into three areas: technological, social and linguistic [2]. Obviously, they are overlapping and interconnected and are only divided for the purpose of our study.

At the technological level, we have some technological ways of organizing speech, messages, and ultimately, our thinking and perception: the use of hashtags, highlights, restrictions in the number of words, restriction in the time (length of videos), and concise messages. All these technically shape our thinking, speech and perception.

At the social level, information is shared in a new way: we deal with information streaming, shaping of individual preferences, the emergence of information bubbles, data collection, big data, the rise of new (often very young) influencers, the restructuring of trading and marketing schemes, new forms of social interaction such as "challenges", and many other manifestations.

At the linguistic level, new (or redefined) terms and concepts come into global use. Among the recent trends are such concepts as abuse, gaslighting, body positive and others. The global, universal concepts are on the rise and come to national mentalities as "ready-to-use" concepts.

One of the most influential features of the new global digital communicative sphere is the remarkable intensity of the information content. At the moment, it is not difficult to observe how significant the production, consumption and distribution of information content has become, but how communication technologies affect the public consciousness as a whole has not yet been sufficiently studied. It is obvious that today there is a shift in the ways of communication: from mass communication (which was established by the mass media) to personalized one (which is promoted by digital platforms). In this shift, the control over content may not be as important as the knowledge of the interests of users, their preferences, and the technical infrastructure to initiate the user involvement in certain types of content.

In many cases, the audience becomes the main target of what is published. Currently, the involvement of the audience is a key marker of information authority and is the main value. As a result, there are changes in not only the type and range of content created and consumed, but also in the formation of special value attitudes and, consequently, a special type of mentality.

Thanks to technological innovations, the consumption of information has been changed dramatically. The path of information dissemination is becoming wider and wider. However, despite all this abundance, consumption patterns and habits are not still well understood. As Joelle Swart writes, "the modern information landscape is characterized by an abundance of digital media and expanded opportunities for users to navigate the news independently. However, it is still unclear how people perceive this changing environment, how they decide which media to choose and which to ignore" [3]. For today's consumers, information is accessible, simplified and often free of charge. It is often individualized and personalized. These properties of information certainly contribute to the formation of a personal mentality, which is reflected in the national consciousness and the mentality of representatives of a certain culture.

According to the theory of Leslie Alvin White, the ability to attach symbolic meaning to thoughts, actions or objects, as well as the perception of symbols, is inherent only in a person [4]. Leslie White's concept of culture includes three components: ideological, social (where collective behavior and its types are considered) and technological, where he considered the latter as the basis for the emergence and development of culture. Leslie White suggests studying the concept of culture through three principles: historical (the historical approach affects temporary processes, that is, the sequence of some historic events); functional (functional analysis exists to study the functional and structural aspects of the development of culture) and evolutionary (i.e. the interpretation of formal-temporal processes, which implies phenomena that represent a temporary sequence of forms).

The advantages of the global digital environment in the formation of a special type of thinking, global thinking, are numerous. Digital platforms provide access to a significant audience, while search engines and social networks are engaging a global audience at an unprecedented speed. Indeed, consumers can now become producers, as evidenced by the appearance of such terms as "prosumer" and "producer". Digital platforms usually do not produce news content. 
However, they play a key role in the dissemination of information, and determine the language means in the formation of the information flow. Thus, they are not so much content producers as simple intermediaries. They perform a hybrid role, which includes, to some extent, both distribution and decision-making about what is and is not an acceptable content, in particular language, which is especially noticeable in social networks.

The language, following the basic ideas of $\mathrm{F}$. de Saussure, is a system of signs expressing ideas; it can be localized where an auditory or visual image is associated with a concept. This is a system of signs in which the only essential thing is the combination of meaning and an acoustic image, and both of these elements of the sign are equally psychic... Associations cemented by collective consent, the totality of which constitutes language, are realities that have their location in the brain. Thus, the digital communicative sphere affects not only the choice of information, but also the choice of language means. In 1989, philosopher Jurgen Habermas perfectly formulated the idea of the public sphere as a place that is fair and accessible to everyone. The concept of the public sphere has been used and adapted by a number of media scientists, including from such disciplines as law, media, journalism and related fields of research. Together, the audience and digital platforms are rebuilding the public sphere, affecting the language environment, among other things. The consequences are ambiguous and still manifest themselves. All these shifts are dramatic, and the pace of change shows no signs of slowing down. The Reuters Research Institute notes in its digital news report for 2020 that "Nothing is holding up - new technologies such as voice interfaces and artificial intelligence are on the way, offering new opportunities, but also new challenges for audiences, regulators and media companies. The future of news remains uncertain". In this uncertain communication environment, digital platforms and consumers of information are responsible for adaptation and change.

The migration of information content to digital channels and the accompanying atomization of information caused the transition from mass communication to personalized and individualized information consumption. Personalization in this context is a digital process that involves searching, sorting and recommending news content based on the explicit and / or implicit preferences of individual users. Customization refers to the modification of the sources, delivery and frequency of digital content for individual consumption. Both personalization and individualization help to filter the abundance of digital information and present information adapted to the interests of a particular person.

Opportunities for personalization and individualization of information consumption have become possible thanks to the growth of online access to news. News available through digital channels has surpassed traditional channels, with social networks, online news platforms and search engines playing a leading role. As a large and still growing proportion of consumers rely on digital platforms for their news, recipients are increasingly dependent on algorithms for "offline" selection of the information content they consume. Such algorithms are used by both digital platforms and traditional mass media.

Digital platforms and digital information producers rely on "recommendation systems" [5] to filter information content. Such systems of prioritization and personalization of information are based on the fixed or assumed preferences of users. The following recommendations are intended to help the user make a decision about the consumption of material. As a rule, recommendation systems work in a cyclical process, which includes:

1) collecting information about the user - the system receives explicit and / or implicit feedback about the user's preferences to build a profile (or model) that is used for forecasting;

2) processing and training - the system applies an algorithm for "training" based on feedback data collected about the user, and adapts the user profile (or model);

3) and forecasting or recommendation - the system then predicts or recommends information content that the user may prefer. This can either be predicted based on the user's behavior on the platform (for example, using probabilistic inference), or recommended directly based on a set of explicit feedback data provided by the user.

The ability of consumers to customize their information flow is associated with the personalization practiced by digital platforms and mass media. Customization, as noted earlier, refers to the modification of the sources, delivery and frequency of digital info content for individual consumption.

To maximize engagement, consumers are increasingly filtering their content to identify narrow, personalized interests. As we have already seen, consumers are increasingly relying on algorithmic systems to provide them with information that matches their preferences. And when digital platforms get an incentive to show content that optimizes interaction, consumers can find themselves in algorithmically constructed "information bubbles". These filter bubbles are personalized according to the user's preferences, and then further enhanced by customization. These cycles of personalization and individualization reinforce the tendency of people to consume news that corresponds to their existing worldviews, which, thus, creates "echo chambers". Potential consequences include limited public discourse, a less informed population, and heightened political polarization.

Diversity is often seen as a fundamental principle of information quality, which helps to ensure a wellinformed population. The balance of info can include a variety of sources, content, and perspectives. However, algorithmic filtering methods involve the risk of limiting diversity, which can lead to information blindness of consumers. This has led scientists to question whether algorithmic methods on digital platforms value and promote diversity as a key characteristic of news quality. Further consequences of filtering effects are that consumers can artificially strengthen their preferences and prospects. "Echo chambers" can cause people to 
completely avoid important public issues, or they can polarize public discussion and, thereby, hinder constructive discussions. It should be noted here that algorithms do not necessarily limit diversity. In fact, the opposite may be true: a variety of content can be programmed into the algorithms of platforms. The effect of an algorithm on diversity depends on the specifics of this algorithm. In addition, various studies on "information bubbles" and "echo chambers" have not provided convincing evidence that algorithmic methods hinder the diversity of content [Dubois, E. \& Blank, 2018; Guess, 2018; Haim, Graefe and Hans-Bernd, 2018; Möller, 2018].

Algorithms can filter and prioritize information in much the same way as editors in a newspaper. Instead of replacing people in content production, algorithms can thus be seen as helping in curating and transmitting some content. However, it can work both ways: promote or limit diversity.

The Internet space is characterized by radical innovations and technological changes. Both digital platforms and recipients of information use the same technologies, including algorithms, personalization, recommendation systems and artificial intelligence. Any assessment of the impact of technology on the language environment should be related to the preliminary question: how much responsibility for user behavior should be assigned to the technology, and how much to the users? The prevailing scientific point of view asserts that users are free, but only within the parameters and values embedded or encoded in the technology [6]. From this point of view, users and technologies influence each other. As sociologist Manuel Castells writes, there is a "dialectical interaction between society and technology" [7]. Therefore, the technology bears some responsibility for the behavior of users. Similarly, legal scientist Alice Marwick recognizes the impact of platform technology on people's behavior. As Castell writes: "platforms do play a role: the material capabilities of technology enhance or suppress certain types of human behavior." She notes, for example, that network television is much more attached to the dominant ideologies than YouTube [7]. The term "affordances" used by Marwick is common in a new field of media and communication research known as "platform research" [Gillespie, 2016; Just \& Latzer, 2017; Nielsen \& Ganter, 2018]. Special attention in this study is paid to how the "availability" of various digital technologies affects people's behavior.

For example, in 2015, Twitter replaced the "star" button with the "heart" button and thereby changed the way users interact with "favorite" content. Although this change may seem insignificant, some users have publicly expressed their outrage. This shows how functions such as the "star/heart" button create some kind of meaning: a function is clearly not just a function. The symbols and the connotations they carry have meaning. Clicking a button means something; it creates different possibilities. While this platform change may seem trivial, it also shows how small features are objects of intense feelings. Another example: Facebook has a "like" button, which was supplemented in 2016 with "love", "haha", "wow", "angry" and "sad", but there is no "hate" button.
We should also take into account the fact that civilization cannot exist without effective communication, because it is difficult to imagine how modern communication, international trade or cultural exchange could exist without them. It is obvious that the world is becoming a single whole, a "single entity" divided by time zones rather than natural geographical boundaries, and this "single entity" correlates with Marshall McLuhan's statement about the creation of a "global village". McLuhan believed that the use of new means of communication in people's lives would not only bring changes in the way people communicate with each other, but also affect all spheres of human activity. $\mathrm{He}$ believed that before the invention of writing, a person lived "in an acoustic space that has the main character of a sphere, whose focus or center is simulated everywhere, and the border is nowhere" [8]. This is a space "without a direction, without a horizon" [8]. McLuhan argued that a person is aware of all his feelings at the same time at any moment of communication. Therefore, when a person tries to communicate through any medium, the possibility of receiving and transmitting information with appropriate accuracy depends on the ability of the environment to include all the relevant human feelings. A person refuses an acoustic space for the sake of a visual one. Any attempt to transfer this unique experience from one person to another requires both distortion and simplification. As a result, the 21st century, the age of information, instant search and total involvement, is a time characterized by the fact that it has means of stability, on the one hand, far surpassing everything that is possible for a visual or civilized and fragmented world; on the other hand, it leads to the simplification of human consciousness. In this aspect, McLuhan's idea is traced that new technologies of the electronic future are taking humanity back to the "caves of the past", where people worship the objects of their own inventions. According to McLuhan, if people do not understand the nature of the new electric mass media, they risk losing all the traditional values of literacy and civilization [8] due to the fact that information is distributed in the "global village" at such a speed that every human action or event will take into account the factor of total involvement of each individual in the life and actions of all mankind. In the era of automation and electricity, the globe is becoming "a community of continuous communication, a single campus in which everyone, regardless of age, is involved in the process" [8]. In this "global village", where communication is continuous, and participation in human dialogue is partial, "the problem of development is to expand consciousness itself" [8].

McLuhan's ideas about the "global village" can be most clearly observed in journalism. Journalism does not stand still, it was changing even before the advent of digital platforms, but digital platforms had a significant impact on the production of information, and journalism had no choice but to adapt. First of all, journalists perceive audience engagement as the main value, which has both positive and negative consequences. One of the consequences is that shorter and more emotional content 
has a much greater prevalence. Digital platforms have also had a significant impact on information consumption. Consumers are increasingly getting access to their news through social networks. Faced with unprecedented choice and convenience, today's consumers are used to receiving information "for free", and in many cases consumers can become part of the distribution and production process. They can share information, react and comment. They can create content. Of course, the individual characteristics of the addressee's mentality, as well as national and cultural ones reflected in the language (in the choice of language units and graphic symbols) affect the quality of information [9]. Since the choice of language means of transmitting information is simplified, and the process of transmitting information occurs instantly, one can observe a "chain reaction" of language simplification. However, the impact of technologies is complex. It is obvious that with the fundamental, ongoing changes in the information landscape, language distinctive features become blurred, thereby influencing the formation of a modern type of thinking, global thinking, and, accordingly, mentality.

Thus, the study of how technological features and algorithms shape the ways people communicate eventually takes us to the issues of values and cultural codes. The issue is crucial today as evidence is present that at the core of the national well-being is citizens' consciousness, the quality of their cultural behavior and the cultural patterns of the community. The new biological threat that we are dealing with today is a prime illustration of the idea. Communities all over the world have seen how much the prevention of the spread of the new infections depends on the conscious behavior of citizens. The readiness to undergo certain restrictions for the safety not only for oneself, but for the others - to wear masks and be put in quarantine - comes from culture patterns as only a cultural person can restrain and restrict themselves for the needs of the other.

The discourse that is employed in governmental attempts to control the social behavior in order to avoid epidemics is expectedly aimed at and structured around cultural values such as responsibility, conscientiousness, the recognition of the value of the human life and health. Obviously, administrative restrictive measures have also been taken; however, they are not fully efficient and put the society at risk of the abuse of such measures that threatens civil liberties.

\section{Conclusions}

The importance of cultural codes and cultural patterns for the national economy is well researched. The impact of cultural patterns on the economic trends is evident at numerous levels. At the macro level, the rise information (digital) economy and a great number of relevant issues such as intellectual property relies upon the existent cultural codes and values. Moreover, a new strong trend is on the rise - minimalism, which can undermine longpersisting consumerism, thus, changing the economy and changing the cultural outlay. Another argument, at the meso level, for the closer interrelation between today's economy and culture is that the production is much under the dictate of the trends - brands choose customization over mass production; take into consideration the trend for ecology. The ways people are buying and consuming are very much more about the culture than the material products. This is especially evident in the restaurant industry - coffeeshops, cafes, restaurants focus heavily on the philosophy, the attitudes, the ideas, and carefully construct their strategies in accordance with their customers' collective (group) identities. At the micro level, spending and managing finances remains one of the few fields (together with health and appearance issues, for instance), in which an individual still finds the freedom to build up their identity. These are the areas that are still under personal control whereas other spheres such as career and political influence are floating more and more out of reach for the people, which is caused by global job competition, the rise of transnational corporations and the globalizing world, in general.

The above-mentioned ideas of the impact of culture on the physical survival and the economic well-being of the nation are just a few of the many ways in which culture is important. However, culture itself is shaped by political, economic and social circumstances. According to Russian economist A. Auzan, culture is sustainable but it is under the influence of laws, political regimes, certain historic events - culture is an imprint of all this [10]. In this way, cultural codes and patterns change the following variations in global communicative sphere; therefore, it is highly important to mark, monitor, analyze and discuss the mechanisms, algorithms and the overall idea and logic behind the new digital communicative space.

To conclude, the researchers that are engaged in the study of the changes in communicative and cultural codes that are brought about by the forming global digital communicative space have a great advantage: they can see the consolidating space in the making as its rules and algorithms are emerging, adapting and shaping right now. Thus, we have a great opportunity to discover many more regularities in how culture, language and thinking work as well as in the interrelations between them.

\section{References}

1. D. Bolinger, Language - the Loaded Weapon: the Use and Abuse of Language Today (2014) (In English)

2. O. Yu. Verbitskaya, A.V. Chaykisova, E.E. Kalish, L.V. Kulgavova, E.V. Lesnikovskaya, EpSBS, NININS 2020, Changes in African American Identity in the 1960s: Social Mind Transstructural Analysis , 102, 1131-1137 (2020) (in English)

3. J. Swart, C. Peters, M. Broersma, Navigating CrossMedia News Use: Media repertoires and the value of news in everyday life (2017) (In English) 
4. L. White, The Evolution of Culture (1959) (In English)

5. F. Isinkaye, Y. Folajimi, B. Ojokoh, Egyptian Informatics Journal Recommendation systems: Principles, methods and evaluation, 10 (2015) (in English)

6. N. Thurman, S. Schifferes, Journalism Studies, The Future of Personalisation at News, 13, (2012) (in English)

7. M. Castells, The Rise of Network Society (1996) (in English)
8. M. McLuhan, Laws of Media: The New Science (1988) (In English)

9. O. Verbitskaya, E. Lesnikovskaya, N. Panchenko, SHS Web Conf., CILDIAH-2018, Codes' Switching as Strategic Resource of an Addresser in Mass Media Discourse, 50, 01195 (2018) (in English)

10. A.N. Auzan, E.N. Nikishina, Sociocultural economy: how culture influences economy and how economy influence culture (2021) (In English) 